\title{
Accuracy of diagnostic testing in primary ciliary dyskinesia: are we there yet?
}

\author{
Eric G. Haarman ${ }^{1}$ and Miriam Schmidts ${ }^{2}$
}

Affiliations: ${ }^{1}$ Dept of Pediatric Pulmonology, VU University Medical Center, Amsterdam, Amsterdam, The Netherlands. ${ }^{2}$ Human Genetics Dept, Radboud University Medical Center and Radboud Institute for Molecular Life Sciences, Nijmegen, The Netherlands.

Correspondence: Eric G. Haarman, Dept of Pediatric Pulmonology, VU University Medical Center, Amsterdam, De Boelelaan 1117, 1081 HV, Amsterdam, The Netherlands. E-mail: eg.haarmandvumc.nl

0

@ERSpublications

Diagnostic testing in PCD: an appraisal of current and future diagnostic techniques http://ow.ly/VBZKr

Although primary ciliary dyskinesia (PCD) is a rare disease, symptoms like recurrent upper and lower respiratory tract infections are extremely common, especially in the paediatric population. Therefore, an important but complicated task for clinicians is to identify patients suffering from this autosomal-recessive condition among the large cohort of individuals with recurrent respiratory symptoms. However, diagnosing PCD is difficult, as there is no single gold standard and it relies on a combination of complex techniques, only available in few specialised centres worldwide [1-4]. There are no evidence-based guidelines and consequently, diagnostic algorithms vary among centres depending on local expertise.

The article by JACKSON et al. [5] is the first to report on the diagnostic accuracy of the most commonly used diagnostic techniques in PCD (high-speed video microscopy (HSVM), nasal nitric oxide and transmission electron microscopy (TEM)), both when used as single tests and when used in different combinations, in a very large cohort of patients specifically assessed at a national PCD centre (Southampton, UK), which also included "inconclusive" patients. Does this mean that we can finally prepare an evidence-based guideline for diagnostic testing of PCD and that we can provide clinicians in various countries with a solid diagnostic algorithm for PCD? Although the data in the article provide an important first step, some issues remain to be addressed.

Similar to the sweat test in cystic fibrosis, HSVM remains an important cornerstone in the diagnosis of PCD, as shown by JACKSON et al. [5] and others [1, 3, 6-8]. This test has both excellent sensitivity and specificity. However, as all who are involved in evaluating ciliary biopsies know, ciliary beat pattern can vary considerably within a single biopsy. In addition, beat patterns are heavily influenced by respiratory tract infections. Even in patients without underlying PCD, beat patterns can remain abnormal for up to 6 weeks after an infection (secondary dyskinesia). Extensive investigator training can help to learn how to discriminate primary from secondary dyskinesia to some extent. However, repeated biopsies are often necessary and even then, contradictive findings are sometimes observed. These problems can be resolved by routine use of cell cultures [9-11], but these tests are available in even fewer centres. In addition, reported success rates of cultures vary considerably (from $54 \%$ to over $79 \%$ ) $[9,12]$, the tests are time-consuming (up to 8 weeks) and costs are relatively high.

In addition, more recently PCD variants have been described with very subtle motility defects, such as those associated with mutations in GAS8 [13]. Likewise, novel mucociliary clearance disorders associated with a reduced number of normal functioning cilia caused by mutations in CCNO [14] and MCIDAS [15] genes producing a clinical respiratory phenotype similar to PCD may be missed by HSVM, even if cell

Received: Nov 162015 | Accepted: Nov 232015

Conflict of interest: None declared.

Copyright @ERS 2016 
culture is performed, as lack of cilia is often wrongly considered secondary to infections or a culture artefact.

Many have advocated the use of nasal nitric oxide as a screening test for PCD [16-19]. Nasal nitric oxide is generally low in PCD patients and can thus be used to discriminate this condition from other diseases leading to a similar respiratory phenotype. The test is widely available, easy to perform and relatively cheap $[16,20]$ However, as is shown by the data from JACKSON et al. [5], up to $10 \%$ of the patients have nasal nitric oxide levels within the normal range. Excluding patients with normal nasal nitric oxide levels from further diagnostic testing would thus lead to an unacceptably high percentage of patients being missed.

Similarly, it has been clear for many years now that TEM can show a (nearly) normal ciliary ultrastructure in specific PCD subtypes [13,21,22]. The current study confirms this by showing that in approximately one fifth of PCD patients seen in their clinic TEM appears to be normal. This means that TEM is also an unreliable tool to exclude PCD if used in isolation. However, if used by adequately trained personal, it appears to have $100 \%$ specificity. Recently, electron tomography has evolved as a research tool enabling three-dimensional visualisation of the ultrastructure of cilia. This technique has demonstrated ultrastructural defects in PCD patients with HYDIN mutations, who did not appear to have defects on classic TEM [21]. Although there are still some limitations to this technique, such as microscopic resolution, limited penetration depth and the speed of data processing, with the current progress in both hardware and software development, we can hope that sensitivity of TEM will further increase in the future and make PCD diagnosis for more difficult cases possible as well.

Jackson and co-workers did not include all diagnostic techniques that are available to date. Recently, immunofluorescence labelling has become available [23]. For immunofluorescence labelling diagnosis, specific antibodies are used to identify proteins that are normally part of the ultrastructure of the cilium in human respiratory epithelial cells and can be absent, mislocalised or present in reduced amounts in PCD patients. Although immunofluorescence labelling has predominantly been used in PCD research in the past, an increasing number of centres are using it as part of their diagnostic work-up. This technique holds great promise, as it is relatively cheap, fast and easy to use, and provides the possibility of identifying abnormal ciliary protein composition or even the underlying molecular defect at a protein level [24]. Immunofluorescence labelling also offers the possibility to identify patients with apparently normal TEM. Therefore, it would be interesting to establish the sensitivity and specificity for this technique as well, both when used alone and when used in combination with the other diagnostic tools available.

Genetic testing was not included in the diagnostic algorithm. So far, $>30$ disease causing genes have been described for PCD and mucociliary clearance disorders. Thanks to the development of high throughput next-generation sequencing technologies in the past few years, mutations can now be identified in up to $70 \%$ of cases. However, the specificity of genetic testing is very high and genetic testing can identify patients missed, for example, by TEM such as patients with mutations in DNAH11 [22], HYDIN [21] and GAS8 [13]. As in $\sim 30 \%$ of cases no mutations can be identified, genetic testing cannot be used to exclude the diagnosis of PCD at the moment.

In summary, the article by JACKSON et al. [5] is an important step towards evidence-based guidelines for PCD diagnostics, including the most commonly used techniques like nasal nitric oxide, HSVM and TEM. But we are not there yet. The work emphasises the importance of HSVM as a technique with relatively high sensitivity and specificity. However, more recently described defects with minor abnormalities in ciliary beat pattern or reduced generation of normal cilia, can easily be missed. Techniques like nasal nitric oxide and TEM are valuable tools for confirming the diagnosis of PCD, but one should be cautious to exclude the diagnosis in the case of normal findings, which contrasts with current practice in many centres. Newer techniques, like genetics and immunofluorescence labelling, hold great promise for the future. As with all clinical dilemma's, the clinician should remain critical in cases of high clinical suspicion and be willing to re-evaluate all diagnostic steps.

\section{References}

1 Leigh MW, O'Callaghan C, Knowles MR. The challenges of diagnosing primary ciliary dyskinesia. Proc Am Thorac Soc 2011; 8: 434-437.

2 Kurkowiak M, Ziętkiewicz E, Witt M. Recent advances in primary ciliary dyskinesia genetics. J Med Genet 2015; 52: $1-9$.

3 Lucas JS, Burgess A, Mitchison HM, et al. Diagnosis and management of primary ciliary dyskinesia. Arch Dis Child 2014; 99: 850-856.

4 Werner C, Onnebrink JG, Omran H. Diagnosis and management of primary ciliary dyskinesia. Cilia 2015; 4: 2.

5 Jackson CL, Behan L, Collins SA, et al. Accuracy of diagnostic testing in primary ciliary dyskinesia. Eur Respir J 2016; 47: 837-848. 
6 Schwabe GC, Hoffmann K, Loges NT, et al. Primary ciliary dyskinesia associated with normal axoneme ultrastructure is caused by DNAH11 mutations. Hum Mutat 2008; 29: 289-298.

7 Amirav I, Mussaffi H, Roth Y, et al. A reach-out system for video microscopy analysis of ciliary motions aiding PCD diagnosis. BMC Res Notes 2015; 8: 71.

8 Barbato A, Frischer T, Kuehni CE, et al. Primary ciliary dyskinesia: a consensus statement on diagnostic and treatment approaches in children. Eur Respir J 2009; 34: 1264-1276.

9 Hirst RA, Jackson CL, Coles JL, et al. Culture of primary ciliary dyskinesia epithelial cells at air-liquid interface can alter ciliary phenotype but remains a robust and informative diagnostic aid. PLoS One 2014; 9: e89675.

10 Hirst RA, Rutman A, Williams G, et al. Ciliated air-liquid cultures as an aid to diagnostic testing of primary ciliary dyskinesia. Chest 2010; 138: 1441-1447.

11 Jorissen M, Willems T, Van der Schueren B, et al. Secondary ciliary dyskinesia is absent after ciliogenesis in culture. Acta Otorhinolaryngol Belg 2000; 54: 333-342.

12 Jorissen M, Willems T. Success rates of respiratory epithelial cell culture techniques with ciliogenesis for diagnosing primary ciliary dyskinesia. Acta Otorhinolaryngol Belg 2000; 54: 357-365.

13 Olbrich H, Cremers C, Loges NT, et al. Loss-of-function GAS8 mutations cause primary ciliary dyskinesia and disrupt the nexin-dynein regulatory complex. Am J Hum Genet 2015; 97: 546-554.

14 Wallmeier J, Al-Mutairi DA, Chen C-T, et al. Mutations in CCNO result in congenital mucociliary clearance disorder with reduced generation of multiple motile cilia. Nat Genet 2014; 46: 646-651.

15 Boon M, Wallmeier J, Ma L, et al. MCIDAS mutations result in a mucociliary clearance disorder with reduced generation of multiple motile cilia. Nat Commun 2014; 5: 4418.

16 Leigh MW, Hazucha MJ, Chawla KK, et al. Standardizing nasal nitric oxide measurement as a test for primary ciliary dyskinesia. Ann Am Thorac Soc 2013; 10: 574-581.

17 Marthin JK, Nielsen KG. Choice of nasal nitric oxide technique as first-line test for primary ciliary dyskinesia. Eur Respir J 2011; 37: 559-565.

18 Collins SA, Gove K, Walker W, et al. Nasal nitric oxide screening for primary ciliary dyskinesia: systematic review and meta-analysis. Eur Respir J 2014; 44: 1589-1599.

19 Walker WT, Jackson CL, Lackie PM, et al. Nitric oxide in primary ciliary dyskinesia. Eur Respir J 2012; 40: 1024-1032.

20 Walker WT, Liew A, Harris A, et al. Upper and lower airway nitric oxide levels in primary ciliary dyskinesia, cystic fibrosis and asthma. Respir Med 2013; 107: 380-386.

21 Olbrich H, Schmidts M, Werner C, et al. Recessive HYDIN mutations cause primary ciliary dyskinesia without randomization of left-right body asymmetry. Am J Hum Genet 2012; 91: 672-684.

22 Knowles MR, Leigh MW, Carson JL, et al. Mutations of DNAH11 in patients with primary ciliary dyskinesia with normal ciliary ultrastructure. Thorax 2012; 67: 433-441.

23 Omran H, Loges NT. Immunofluorescence staining of ciliated respiratory epithelial cells. Methods Cell Biol 2009; 91: 123-133.

24 Fliegauf M, Olbrich H, Horvath J, et al. Mislocalization of DNAH5 and DNAH9 in respiratory cells from patients with primary ciliary dyskinesia. Am J Respir Crit Care Med 2005; 171: 1343-1349. 